\title{
EDGE FEATURE MATCHING OF REMOTE SENSING IMAGES VIA PARAMETER DECOMPOSITION OF AFFINE TRANSFORMATION MODEL
}

\author{
Chen Tianze ${ }^{\mathrm{a}, *}$, Gongjian Wen ${ }^{\mathrm{a}}$, Zhongliang Jiang ${ }^{\mathrm{a}}$, Bangwam Yin ${ }^{\mathrm{a}}$ \\ ${ }^{a}$ School of Electronic Science and Engineering ,National University of Defense Technology, Changsha 410073, China
}

Commission VII , WG VII/6

KEY WORDS: Edge Feature Matching, Affine Transformation Model, Parameter Decomposition, Pixel Migration, GA

\begin{abstract}
:
An approach of the edge feature matching of remote sensing images based on parameter decomposition of the affine transformation model is presented. First, the adaptability of affine transformation model used in image matching is analyzed, and parameters of the affine transformation model are decomposed. Second, the sum of squared gradient (SSG) similarity is constructed based on the edge feature in the images, and the parameters of the affine transformation model between the images are solved with the Genetic Algorithms (GA) which is used to obtain the global optimum solution of the similarity. Finally, the performance of the approach is analyzed in theory and validated with remote sensing image matching experiments.
\end{abstract}

\section{INTRODUCTION}

The aim of remote sensing image matching is to find the corresponding alignment relationship between the remote sensing image pair within the same scene, which is acquired from different time, different views or different sensors. It is the kernel of many remote sensing applications, such as 3D reconstruction, target recognition, matching guidance, change detection and data fusion. Affected by the imaging time, pose, type of sensors, and image noise, image magnitude of the same ground scene indifferent images are hardly identical. In order to improve the robustness, adaptability, and feasibility of matching with multimodalities images, the feature matching method is often used in image matching practically. Popular matching methods include dichotomy relevance method, distance transformation method, structure matching method, chain code correlation method, invariant moment method, and so on(Zitova B, Flusser J,2003).

Presently, matching features are mostly geometry features, such as points, lines, contours and skeletons. The structural feature represented and described by edge feature is the primary component of the image. Edge structures of two images within the same ground scene mostly represent the physical characteristics coexisted in the same scene. In a manner, they are similar to each other. Hence, the image matching can be accomplished between edge sets which include structural characteristics of the image pair.

Feature extraction and description are the fundamental steps for remote sensing image feature matching. At present, most of the traditional edge feature matching methods need feature description with complicated methods of edge points grouping. The precondition of these matching methods, such as line feature matching after collinear edge points grouping(Schmid C, Zisserman A,1997; S.Noronha, R.Nevatia, 2001; Yasser Almehio, Samia Bouchafa,2010), triangulated network matching after the triangulated networks constructed with the relationship among the edge points(Xin Kang, Chongzhao Han, Yi Yang,2006), edge histogram matching with edge statistic histograms constructed among the edge points(Jun Xie, PhengAnn Hengb, Mubarak Shah,2008), is that the corresponding feature must be coexisted in the two images and could be extracted exactly. The image pair with unclear matching features will match unsuccessfully. Moreover, those images, in which the corresponding features coexist, could be mismatched easily yet. Because the feature extraction method may be complicated and the feature description may be affected by methods such as edge grouping, triangulated networks building and histogram construction. The corresponding features can hardly be extracted from the two images. This leads the matching method to be delicate and inadaptable. That is to say, image matching method with high performance should use the features which are coexisted in the image pair, extracted and described with the simple method and progress.

Based on the idea, Yosi Keller (Yosi Keller, Amir Averbach, 2003, Yosi Keller,2006 ) and Jianchao Ya(Jianchao Yao, Kian Liong Goh,2006) presented a matching method of multi-sensor images, which use the pixel migration method to construct the SSG similarity of edge features. The edge features generally exist in the image of all kinds of scene and target. Compared with the other methods, the obvious advantage of the method is that the feature descriptor is simple, and it only needs a brief extraction of edge feature. The adaptability of the matching method can be improved.

However, it leads to another typical difficult, that is the corresponding feature matching, which solves transformation model parameters, must be realized by the searching in the global parameter space. Because it cannot use the geometry invariant directly, or take into account the relationship (such as geometry relationship) of edge sets between the two images to reduce the parameter's range. Therefore, the image matching problem has been turned into a mathematical optimization problem.

As to optimization searching method, the construction of parametric space is crucial to the optimization precision and efficiency. In spatial transformation model of the image matching, the six parameters of the traditional affine transformation model are all solved directly, which may cause low matching precision. Moreover, most of the parameters do not have the clear geometric meaning. The parameter's range, resolution and threshold cannot be concluded validly, and the searching of the image matching may not be convergent, or the low speed of the convergence decreases the matching efficiency. To solve the question, an edge feature matching approach of remote sensing images based on the parameter decomposition of affine transformation model is presented. First, parameters of the affine transformation model are decomposed, the geometric meaning of each parameter is explicit and the range of each parameter can be determined validly. Second, the differential operator and the Ratio of Exponentially Weighted Averages (ROEWA) detector are used to extract the edge

\footnotetext{
* Tianze Chen: tzchen77@ 126.com. Subject of funding of the National Natural Science Foundation of China (61002023)
} 
features from each kind of RS images, and the SSG similarity is constructed based on the edge features. Finally, parameters of the model are solved with the GA which is used to obtain the global optimum solution of the similarity. Thus, the adaptability, the precision and the efficiency can be improved.

\section{EDGE FEATURE MATCHING BASED ON PIXEL MIGRATION}

\subsection{Pixel Migration Algorithm}

The geometry features, which are often used in the image matching (such as point, line, shape, contour, skeleton), are always rooted in the image regions with sharp edge sets. These edge sets with the high gradient magnitudes are combinations of a heap of different structural features and a few of irrelevant elements (such as noise). These structural features in different images with the same scene are implicit similar.

Pixel migration approach introduces a robust matching criterion by aligning the locations of gradient maxima, initialized from the first image. The alignment is formulated as a parametric variation optimization problem which is solved iteratively using the intensities of the second image (Jianchao Yao, Kian Liong Goh,2006).The edge feature matching method based on the pixel migration can realize robust matching among multiimages with extracting edge sets, constructing a corresponding similarity and using a suitable parameter optimization method. In mathematics, the nature of image matching is a parametric optimization problem of the transformation model. The mathematical model of image matching is:

$$
\min _{f, T} J=\sum_{k}\left[I_{1}(x(k))-f\left(I_{2}(T(x(k)))\right]^{2}\right.
$$

Where $T$ is the transformation model, $I_{1}, I_{2}$ is the image information (such as gray values) of each image, $f \circ I$ is a mapping which represents the image information, $J$ is the similarity norm function. For feature matching, with the extracted corresponding edge features $x_{1}(k)$ and $x_{2}(k)$, the model can be simplified as

$$
\min _{T} J=\sum_{k}\left[x_{1}(k)-x_{2}(k)\right]^{2}, \quad x_{2}(k)=T \circ x_{1}(k)
$$

\subsection{Construction of similarity measure}

Pixels having high gradient magnitudes correspond to primary structures of the image, of which the detection is robust. Contrary to high level geometric primitives such as contours and corners, no preset thresholds are needed(Yosi Keller, Amir Averbach,2003). The matching criterion is the global maxima of SSG which is obtained by the iterative parametric variation optimization process. Those pixels with gradient maxima, which are initialized from the first image, are transformed to another image by pixels migration, each pixel migration corresponds to a set of spatial transformation parameters and an SSG value. Only when the corresponding SSG is the maxima, the pixel migration is valid. Hence, the set of parameters which corresponds to the global maxima of the SSG is the solution. The mathematics model is:

$$
\max _{P} J=\sum_{S_{2} \in I_{2}} \mid \nabla I_{1}\left(\left.S_{2}(P)\right|^{2}\right.
$$

Where $S_{2}$ is a set of pixels with high gradient magnitudes in $I_{2}$, $\mathrm{P}$ is the parameter vector for the spatial transformation model, $\left|\nabla I_{1}\right|$ is the gradient magnitudes in $I_{1}$. The matching process can be summarized as an iterative parametric variation optimization process to get the maxima of the SSG in $I_{1}$. The optimization process is initialized with the locations of gradient maxima in $I_{2}$. The matching process is:

Step1.Selecting $S_{2}$ as edges set with high gradient magnitudes in $I_{2}$;

Step2. Constructing $S_{1}$ as edges set in $I_{1}$;

Step3. Constructing $S_{1}(P)$ as edges set, locations of these pixels in $I_{1}$ are the spatial transformations of $S_{2}$ with model parameter vector $\mathrm{P}$;

Step4. The optimization objective function is:

$$
F\left(S_{1}(P)\right) \triangleq \sum_{\left(x_{i}, y_{i}\right) \in S_{1}(P)}\left|\nabla I_{1}\left(x_{i}, y_{i}\right)\right|^{2}
$$

Step5. Parametric optimization to get the maxima of the SSG in $S_{1}$.

The proposed scheme is illustrated in Figure 1.

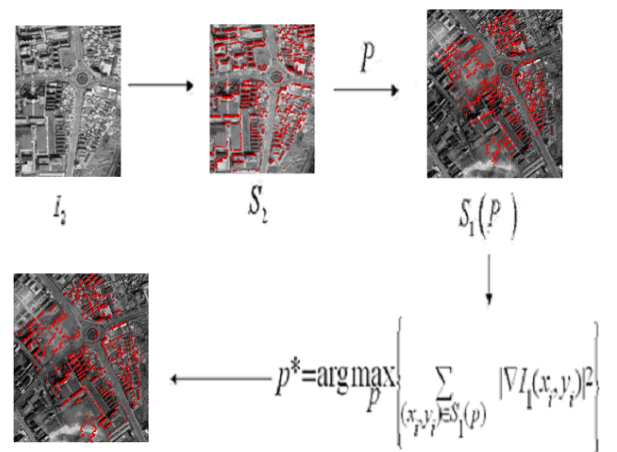

Figure 1.The sketch map of matching algorithm

\subsection{Edge feature extraction of remote sensing Image}

The structural features are much more robust than the gray information in remote sensing images. The similarity computed directly from the original gray image may lead to unsuccessful matching. So, the similarity is constructed with the edge map in this paper.

2.3.1 Edge Feature Extraction of Optical Image: The differential operators are adopted to extract the edge features from the optical image. The traditional differential operators include Sobel operator, LOG operator, etc.

2.3.2 Edge Strength Extraction of SAR Image: Speckle is the inherent property of SAR image. Affected by the speckle, extraction of edge features from SAR images especially needs to take account of the statistical properties of speckled SAR images. Because the speckle preserves the multiplicative model for data distribution, the differential grads edge detectors, which perform well in optical images and depend on the hypothesis that the image is stained by the additive noise, cannot be used in SAR images (Jia Chengli,2006). Ratio of Averages (ROA) detector(Touzi R, Lopes A, Bousquet P,1988) designed by Bovik and ROEWA algorithm(Fjørtoft R, Lopès A, Marthon P, et a,1988)presented by Fjortoft's group are the main methods of edge detection in SAR image. The multi-edges model which used by the ROEWA algorithm is more suitable for the practical SAR image than the others. Moreover, the ROEWA algorithm has commonness with the differential edge detector. The most important is that, in the edge extraction result, a high value indicates the presence of an edge and a low value indicates the absence of an edge. So the edge intensity map which is yielded by the ROEWA algorithm can be considered as the grad image. 


\section{AFFINE TRANSFORMATION MODEL AND PARAMETER DECOMPOSITION}

The aim of Image matching is to build the geometric relationship betweenen the two images, which needs to construct the mathematic model of the image transformation. The model includes: rigid transformation, similarity transformation, affine transformation, projection transformation, polynomial transformation, and so on. The affine transformation is the most popular transformation model of image matching. Based on the multi-views imaging, it has been proved that the affine model is suitable for the transformation between the images whose sensors are far away from the scene with flat terrain (Chen Tao,2006). For remote sensing image, the ground is far away from the sensor which is carried on the remote sensing platform, the Z-coordinates of the corresponding points or their ratio are nearly a constant when the terrain varies relaxed. That is to say, the remote sensing images transformation can be described by the affine model.

\subsection{Affine Transformation Model}

Except for the geometry transformation of shift, rotation, zooming, the affine transformation model also takes into account the shear ( namely the variety of the aspect ratio, or the zooming along the diagonal )between images.

The formula of the affine transformation model is :

$$
\left\{\begin{array}{l}
x_{2}=a_{1} x_{1}+b_{1} y_{1}+c_{1} \\
y_{2}=a_{2} x_{1}+b_{2} y_{1}+c_{2}
\end{array}\right.
$$

Where $\left(x_{1}, y_{1}\right),\left(x_{2}, y_{2}\right)$ are the coordinates of the pre-matching image and the reference image respectively. $a_{1}, b_{1}, c_{1}, a_{2}, b_{2}, c_{2}$ are parameters of the affine model. Parameters $c_{1}, c_{2}$ express the shift between images, but parameters $a_{1}, b_{1}, a_{2}, b_{2}$ have no explicit geometry meaning. The range of each parameter cannot be determined validly. In order to avoid matching unsuccessfully, the range has to be enlarged and the searching time has to be extended.

\subsection{Model Parameter decomposition}

To clarify the geometry meaning of the parameters $a_{1}, b_{1}, a_{2}, b_{2}$, the affine transformation is turned into four steps which include zooming, rotation, shear and shift. Figure 2 shows the sketch map of the transformation procedure.

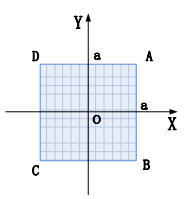

(a)Original image

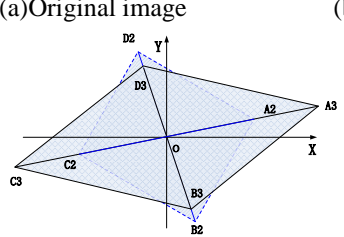

(d) Shear

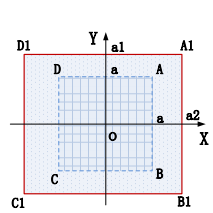

(b) Zooming

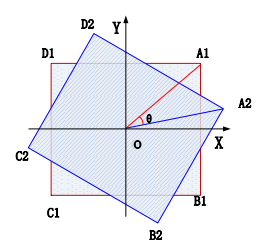

(c) Rotation

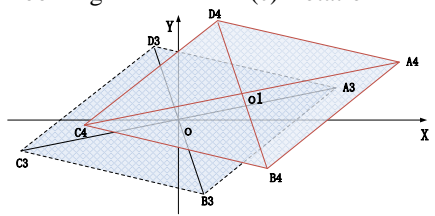

(e) Shift

Figure 2.Sketch map of the affine transformation procedure In Figure 2, the dashed line indicates the graph before the transformation; the real line indicates the graph after the transformation. The horizontal scale, the vertical scale, the rotation angle, the shear scale, the horizontal shift, the vertical shift, are named as $s_{x}, \mathrm{~s}_{y}, \theta, r, d_{x}, \mathrm{~d}_{y}$ respectively. Then parameters of formula (5) can be computed with formula (6).

$$
\left\{\begin{array}{l}
a_{1}=s_{x}(m \cos \theta+n \sin \theta) \\
a_{2}=s_{x}(n \cos \theta-m \sin \theta) \\
b_{1}=s_{y}(n \cos \theta+m \sin \theta) \\
b_{2}=s_{y}(m \cos \theta-n \sin \theta) \\
c_{1}=d_{x} \\
c_{2}=d_{y}
\end{array}\right.
$$

where $m=\left(r+\sqrt{2-r^{2}}\right) / 2, \quad n=\left(r-\sqrt{2-r^{2}}\right) / 2$. With the parameter decomposition, the four parameters $a_{1}, \mathrm{~b}_{1}, a_{2}, b_{2}$ of formula(5) with unclear geometry meaning can be computed by the four parameters $s_{x}, \mathrm{~s}_{y}, \theta, r$ with explicit geometry meaning and valid range. The horizontal scale $s_{x}$ and the vertical scale $s_{y}$ can be approximately estimated with resolution of the two images, and their ranges are often identical to each other. Theoretically, the range is $(0, \infty)$. Commonly, in practical remote sensing image matching, $s_{x}, s_{y}$ is less than or equal to 10 . So the range of $s_{x}, s_{y}$ is $(0,10]$ respectively. The range of parameter $\theta$ is [0,360), the range of parameter $r$ is $(0, \sqrt{2})$. The range of $d_{x}, \mathrm{~d}_{y}$ can be determined specifically with the image size.

\section{OPTIMIZATION OF THE OBJECTIVE FUNCTION}

\subsection{Analysis of the optimization methods}

In fact, the searching of the objective function (4) is a multivariable optimization problem. In image matching, this problem with explicit mathematics structure, high dimension, high computation cost, and multi extreme value, cannot be solved by conventional optimization algorithm (such as the Newton's iterative optimization method, Powell algorithm). The value of SSG is dependent on the variation of the parameter $P$. Figure 3 illustrates the SSG value changed with the parameter $b_{2}$, whose resolution is 0.001 , and the other five parameters are invariable.

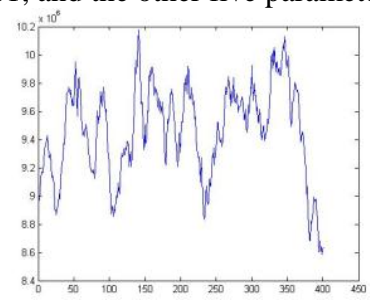

Figure 3. SSG changed by a parameter

Figure 3 shows that the SSG value oscillates sharply with the variation of the parameter $b_{2}$. If the six parameters are all considered, it will be more complex and more difficult to obtain the global optimization. Since SSG may have many local extreme values, the solved parameters set for spatial transformation may not correspond to the global maximum of the SSG surface across the whole searching space. It will converge inherently to some local maximum, if the local optimizer (Newton's iterative optimization method) is used and the initial value is near to the local maximum.GA of the intelligent optimization algorithm can find the global optimization from the initial value along multipath, and it is a valid adaptive optimization algorithm. Hence, GA is used to optimize the parameters in this paper.

\subsection{Optimization of the Genetic Algorithm}

GA is based on the natural selection and evolution of population concept ( $\mathrm{H}$. Holland,1975). It is a parallel random adaptive searching procedure.GA is a simple and robust method 
for optimization problem, and expresses the problem's solution as the procedure of the chromosome's (or individual) survival of the fittest. Each individual represents a solution and is encoded as a chromosome string. The selection, crossover and mutation operators are tuned to preserve the good genetic materials and explore new regions in the searching space. The evolution of individuals in the population is governed by the principle of the survival of the fittest. The fitness of the individual is a function of the decoded chromosome string. With the evaluation of the individual and the operation of the gene, better solutions are evolved and the approximate optimization solution is obtained.

Due to its intrinsic parallelism and the principle of the survival of the fittest in the evolution, in this paper, the SSG is regarded as the fitness of the individual directly, and the fittest individual, i.e. the global optimization solution, is obtained by searching in the parameter space. The degree of diversification is dictated by the choices of the control parameters: number of genes in an individual $\left(N_{g i}\right)$, length (bit numb) of each gene $\left(L_{g}\right)$, length (bit number) of each chromosome $\left(L_{c}\right)$, chromosome size of each generation $\left(S_{c}\right)$, crossover rate $\left(P_{c}\right)$, mutation rate $\left(P_{m}\right)$, and iterative number of generations $\left(N_{g}\right)$.

The optimization procedure is:

(1).Determination of transformation model and the parameter number. The model parameter vector after the model decomposition is: $P=\left[s_{x}, \mathrm{~s}_{y}, \theta, r, d_{x}, \mathrm{~d}_{y}\right]^{T}$;

(2). Partition of the parameter space, determination of each parameter's range and the length of the chromosome;

(3). Extraction and location the initial pixels with high gradient magnitude from the image $I_{1}$;

(4). Determination the coding method, setting the control parameters $\left(N_{g i}, L_{g}, L_{c}, S_{c}, P_{c}, P_{m}, N_{g}\right)$ of GA and construction the fitness function;

(5). Initialization of the chromosome;

(6).Genetic iteration until the end condition is satisfied.

6.1 Offspring selection with the fitness optimization strategy, namely the crossover and mutation operation. The crossover operator is random pairing and the two-point crossover, mutation operator is the gens exploitation according to its statistical probability.

6.2 Chromosome coding, space transformation with the model parameters, SSG computation and the fitness evaluation of the individual.

6.3 Ending until the offspring not evolve any more or iterative generation exceeds $N_{g}$, or else returns to 6.1.

\section{PERFORMANCE ANALYSIS}

The performance of the image matching includes precision, efficiency and robustness.

\subsection{Matching precision}

Deduced form the formula (5) of transformation model between images, in theory, the matching precision is determined by the parametric precision and the image size.

The parametric precision based on the parameter searching space is determined by resolution. In this paper, with the precondition of the acceptable computational load, the resolution of parameters $s_{x}, s_{y}, r$ is $10^{-2}$ order of magnitude, and the resolution of parameters $\theta, d_{x}, \mathrm{~d}_{y}$ is $10^{-1}$ order of magnitude. So resolution of the traditional affine model coefficients $a_{1}, b_{1}, a_{2}, b_{2}$ is $10^{-2} \times\left(10^{-2} \times 10^{-2}\right)$ order of magnitude,viz. $10^{-6}$.
Resolution of coefficient $c_{1}, c_{2}$ is equal to the order of magnitude of parameters $d_{x}, \mathrm{~d}_{y}$, namely $10^{-1}$. Given the order of magnitude of the image size as $10^{\mathrm{x}}$,then, the order of magnitude of final matching precision is $\max \left(10^{\mathrm{x}-6}, 10^{-1}\right)$. Under the same precondition, in the previous methods, resolution of parameters $a_{1}, b_{1}, a_{2}, b_{2}$ is $10^{-3}$ order of magnitude, so the final matching precision is $\max \left(10^{\mathrm{x}-3}, 10^{-1}\right)$.

In theory, when the order of magnitude of the image size is less than or equal to $10^{2}$, the matching precision of the proposed approach and the previous methods can both up to $10^{-1}$ order of magnitude. When the image size is more than $10^{2}$ and less than $10^{5}$ orderof magnitude, the matching precision of the proposed approach can up to $10^{-1}$ order of magnitude, the previous methods can only up to $10^{\mathrm{x}-3}$ order of magnitude. When the image size is more than $10^{5}$ order of magnitude, the matching precision of the proposed approach can up to $10^{\mathrm{x}-6}$ order of magnitude, the previous methods can still up to $10^{\mathrm{x}-3}$ order of magnitude. Commonly, the remote sensing image size is $10^{3}-10^{4}$ order of magnitude, so the matching precision in this paper can up to $10^{-1}$ order of magnitude, and up to $10^{0}-10^{1}$ order of magnitude in methods. So, the matching precision in this paper is 1 to 3 orders of magnitude higher than the previous method's.

Moreover, to realize the more precise matching, the proposed approach only needs to improve the resolution of the parameters $d_{x}, \mathrm{~d}_{y}$ (viz. $c_{1}, \mathrm{c}_{2}$ ) in this paper, yet, it needs to improve the resolution of the whole six parameters $a_{1}, \mathrm{~b}_{1}, a_{2}, b_{2}, c_{1}, \mathrm{c}_{2}$ in the previous methods. It will increase the computational load greatly.

\subsection{Matching efficiency}

The emphasis of the proposed approach lies in searching the optimization SSG in the parameter space. The matching efficiency is basically determined by the computational load of the parameter iterative searching. Using the proposed approach, the computational load can be decreased and the searching efficiency can be improved greatly.

First, in previous methods, since most of the parameters have unclear geometry meaning, each parameter's range cannot be determined exactly in practical matching. In order to maintain the matching robustness, the parameter's range has to be broadened, the number of individuals has to be enlarged and bits of each gene has to be extended, the number of generations has to be increased. Then, the computational load will increase. In this paper, all parameters have explicit geometry meaning, the initial value and the range of each parameter can be determined validly according to the truth. It can decrease the computational load greatly.

Second, the six affine model parameters are all evaluate directly in the previous methods, but are computed by the multiplication operation in this paper. Hence, to maintain the same resolution of the same affine model parameter, the number of individuals can be decreased greatly in this paper. For example, the resolution of the parameters $s_{x}, \mathrm{~s}_{y}, \theta, r$ is 0.1 , known from the formula (6), the resolution of the corresponding four affine model parameters $a_{1}, \mathrm{~b}_{1}, a_{2}, b_{2}$ are 0.01 . So, with the precondition that each parameter has the same range, the chromosome bits in this paper is only $1 / 10$ of the previous methods.

Finally, in practice, the horizontal resolution is equal to the vertical resolution in remote sensing image commonly. So the horizontal scale is equal to the vertical scale, that is to say, the parameters $s_{x}, \mathrm{~S}_{y}$ can be simplified as one parameter. The six 
parameters of the affine model can be reduced to five parameters, the searching space dimension is also decreased.

\subsection{Matching robustness}

On the one hand, the edge feature is used to match image pair in this paper. The method and the process of the edge feature extraction are both simple. The instance that no feature will be found in the image cannot be existed. So the matching feature will definitely be extracted by the proposed approach. On the other hand, GA is used to optimize the parameters, it ensures that the iteration is convergence and the global optimization will surely be found. So the correct matching is found, and instance that unable to match will not be appeared.

\section{EXPERIMENTAL RESULTS}

The proposed approach was experimentally evaluated by image pairs acquired by remote sensors of SAR and optical detector. Both experiments are realized by Matlab simulation. Due to the conventionality that $s_{x}=\mathrm{S}_{y}$ in remote sensing image, in the experiments $P=\left[s_{x}, \theta, r, d_{x}, d_{y}\right]^{T}$. The RMSE is used to evaluate the matching precision. Its definition is:

$$
R M S E=\sqrt{\frac{\sum_{i=1}^{n}\left[\left(a_{1} x_{i}+b_{1} y_{i}+c_{1}-x_{i}^{\prime}\right)^{2}+\left(a_{2} x_{i}+b_{2} y_{i}+c p_{2}-y_{i}^{\prime}\right)^{2}\right]}{n}}
$$

Where, $\left(x_{i}, y_{i}\right) \quad\left(x_{i}{ }^{\prime}, y_{i}{ }^{\prime}\right)$ are the control point's coordinates of the pre-matching image and the reference image, respectively.

\subsection{SAR image matching}

This matching experiment is tested in an aerial SAR image pair with some common overlap to search the corresponding features. Each image is $93 \times 74$ pixels.

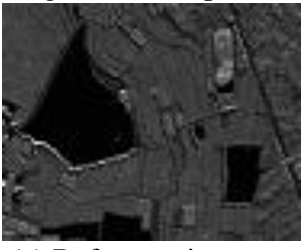

(a) Reference image

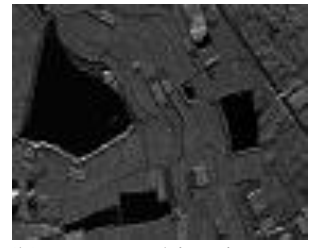

(b) pre-matching image
Figure 4. The original images

The special transformation is used with the affine model. The GA coding is binary Gray code, the bit number of each gene is determined jointly by the parameter's range and resolution. Range and resolution of each gene are showed in Table 1.

TABLE1. RANGE AND RESOLUTION OF EACH GENE

\begin{tabular}{|l|l|l|l|}
\hline Model parameter & Parameter range & $L_{g}$ & Resolution \\
\hline$s_{x}$ & {$[0.7,1.5]$} & 5 & 0.025 \\
\hline$\theta$ & {$[-15,15]$} & 6 & 0.4688 \\
\hline$r$ & {$[0.7,1.4]$} & 6 & 0.0109 \\
\hline$d_{x}$ & {$[-30,30]$} & 7 & 0.4688 \\
\hline$d_{y}$ & {$[-30,30]$} & 7 & 0.4688 \\
\hline
\end{tabular}

initial chromosome is created randomly. The matching results are shown in Figure 5.
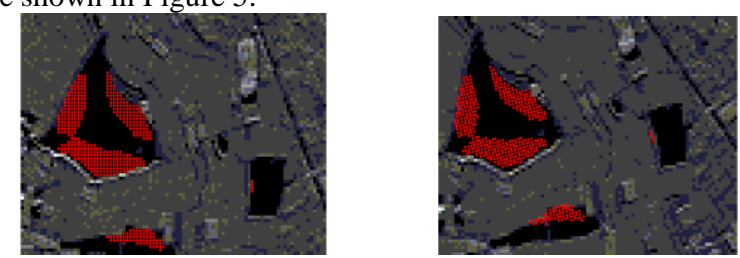

(a)Matching edge set of $S_{1}$ (b)Optimization matching edge set of $S_{2}$

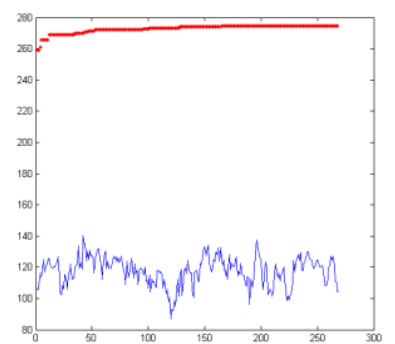

(c) Chart of SSG convergence with the iterative number Figure 5.Matching results

The control points are the corresponding points in the image pair which are collected manually. The coordinates are shown in Table 2.

TABLE 2. COORDINATES OF CORRESPONDING CONTROL POINTS IN THE IMAGE PAIR

\begin{tabular}{|l|l|l|}
\hline NO & $\begin{array}{l}\text { Coordinate of the } \\
\text { reference image }\end{array}$ & $\begin{array}{l}\text { Coordinate of the pre- } \\
\text { matching image }\end{array}$ \\
\hline 1 & $(13,4)$ & $(26,6)$ \\
\hline 2 & $(22,48)$ & $(23,51)$ \\
\hline 3 & $(30,21)$ & $(37,28)$ \\
\hline 4 & $(76,27)$ & $(81,44)$ \\
\hline 5 & $(63,13)$ & $(72,28)$ \\
\hline 6 & $(34,59)$ & $(32,65)$ \\
\hline 7 & $(49,52)$ & $(48,62)$ \\
\hline 8 & $(75,27)$ & $(80,44)$ \\
\hline
\end{tabular}
is $\mathrm{P}=\left[\begin{array}{lllll}0.9839 & 15.0000 & 0.9889 & -13.4646 & 5.4331\end{array}\right]^{\mathrm{T}}$ the matching precision is $R M S E=0.8975$. The matching precision can up to sub-pixel level, it indicates that the proposed approach can be used in many applications. It cost 68.328 seconds to realize accurate matching.

To demonstrate the advantage of the proposed approach, the matching experiment is implemented with the previous methods which do not decompose the parameters. The GA control parameters are: $L_{c}=47, S_{c}=500, P_{c}=0.7, P_{m}=0.08$. When $N_{g}=500, R M S E=1.7074$. The matching precision is nearly twice of the proposed approach. It cost 293.516 seconds, the runtime is almost fourfold of the proposed approach.

\subsection{Matching of SAR and optical images}

This matching experiment is tested in a SAR image and an optical image which have some common overlap to search the corresponding features. The optical image is $150 \times 200$ pixels, the SAR image is $200 \times 350$ pixels. The SAR image's resolution is higher than the optical image's resolution, hence, the optical image is selected as $I_{1}, S_{1}$ is extracted from the $I_{1}$, and SSG optimization searching is realized in the SAR image.

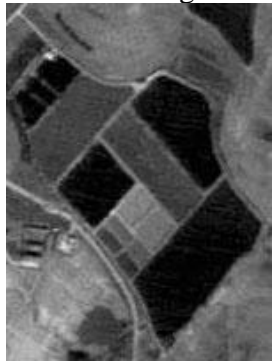

(a) Optical original image

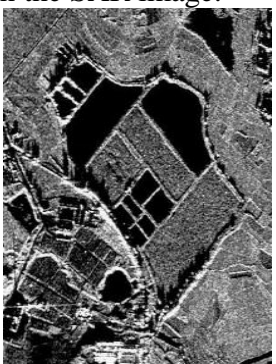

(b) SAR original image
Figure 6. Original images 
The matching flow is the same as the above experiment. The parameter's range and the resolution are showed in Table 3.

TABLE 3. RANGE AND RESOLUTION OF EACH GENE

\begin{tabular}{|l|l|l|l|}
\hline Model parameter & Parameter range & $L_{g}$ & Resolution \\
\hline$s_{x}$ & {$[0.6,1.8]$} & 5 & 0.0375 \\
\hline$\theta$ & {$[-10,10]$} & 6 & 0.3125 \\
\hline$r$ & {$[0.6,1.4]$} & 5 & 0.025 \\
\hline$d_{x}$ & {$[-100,100]$} & 9 & 0.3906 \\
\hline$d_{y}$ & {$[-100,100]$} & 9 & 0.3906 \\
\hline
\end{tabular}

$L_{c}=34, S_{c}=300, P_{c}=0.7, P_{m}=0.08, N_{g}=250$.

The matching results are shown in Figure 7.
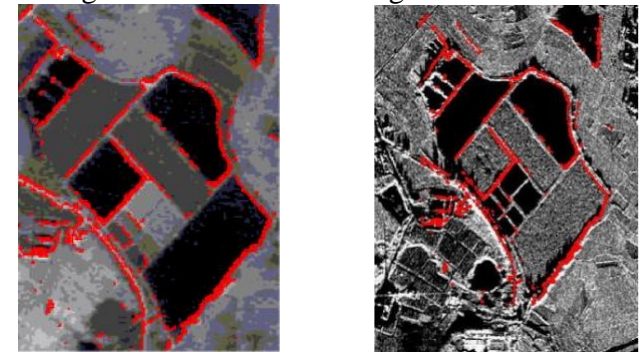

(a) Matching edge set of $S_{1}$ (b) Optimization matching edge set of $S_{2}$

Figure 7.Matching results

Ten control points are collected manually, the coordinates are shown in Table 4.

TABLE 4. COORDINATES OF CORRESPONDING CONTROL POINTS IN THE IMAGE PAIR

\begin{tabular}{|l|l|l|}
\hline NO & $\begin{array}{l}\text { Coordinate of the } \\
\text { optical image }\end{array}$ & $\begin{array}{l}\text { Coordinate of the } \\
\text { SAR image }\end{array}$ \\
\hline 1 & $(6,5)$ & $(56,16)$ \\
\hline 2 & $(44,38)$ & $(115,66)$ \\
\hline 3 & $(51,74)$ & $(127,118)$ \\
\hline 4 & $(68,56)$ & $(150,93)$ \\
\hline 5 & $(11,107)$ & $(216,168)$ \\
\hline 6 & $(95,124)$ & $(195,193)$ \\
\hline 7 & $(61,116)$ & $(140,181)$ \\
\hline 8 & $(71,127)$ & $(153,195)$ \\
\hline 9 & $(82,139)$ & $(171,214)$ \\
\hline 10 & $(75,148)$ & $(162,226)$ \\
\hline
\end{tabular}

$\mathrm{P}=\left[\begin{array}{lllll}1.4774 & 0.1587 & 1.0129 & 48.5323 & 7.4364\end{array}\right]^{\mathrm{T}}$.The matching precision is $R M S E=1.9157$. The runtime is 203.796 seconds.

This experiment is also implemented with the previous methods. The GA control parameters are: $L_{c}=47, S_{c}=500, P_{c}=0.65$, $P_{m}=0.08$ In order to up to the same order of magnitude of the proposed approach, the iterative number has to up to 1150 . The runtime is 1933.015 seconds, it is almost 9.5 times of the proposed approach.

\subsection{Results Analysis}

From the experimental results, the matching precision is lower than the theoretical precision. The main reason is that GA is used as the model parameter optimization searcher. The crossover and mutation operators are optimized randomly, it may result in that the parameter resolution is not the optimization. Moreover, the experimental image size is less than or equal to $10^{2}$ order of magnitude, it cannot fully show the performance of the proposed approach. If the image size is larger, the precision will be more precious. Even though, the matching precision is higher than the previous methods.
Furthermore, the control points are collected manually, each control point's coordinate may include one to two pixels' error. So the matching precision is available to most of the applications. The proposed approach is valid and practicable.

In matching efficiency, the searching of the proposed approach is faster than the previous methods which employ the GA to optimize the model parameters. However, due to the GA's low searching efficiency, it still cost long time to realize image matching.

\section{CONCLUSIONS}

In this paper, the remote sensing image matching is implemented with the SSG similarity based on the edge feature. The experimental results show that the proposed approach can be practicable to the image matching between SAR and SAR, SAR and optical remote sensing images. With the decomposed parameter affine model, the specific parameters are initialized definitely and the resolution is determined explicitly, the complexity of the affine model parameter optimization is simplified greatly. Moreover, the affine model with parameter decomposition can be popularized to those applications which need to optimize the affine model parameters.

Although the efficiency is improved by the parameter decomposition, the optimization time based on the GA is still long. The more efficient convergence algorithm or other optimization methods are needed to improve the efficiency.

\section{REFERENCES}

Chengli Jia. Extraction of Road and Airport in SAR Images. 2006,GraduateSchool of NationalUniversity of Defense Technology,Changsha, Hunan, P.R.China..pp.35-38

Fjørtoft R, Lopès A, Marthon P, et al. 1998,An optimal multiedge detector for SAR image segmentation. IEEE Transactions on Geoscience and Remote Sensing, 36,pp.793-802.

Jianchao Yao, Kian Liong Goh. 2006,A Refined Algorithm for Multisensor Image Registration Based on Pixel Migration.IEEE Transon Processing. 15,pp.1839-1847

J. H. Holland, 1975 Adaptation in Natural and Artificial System. Ann Arbor, MI: Univ. Michigan Press.pp.56-58

Jun Xie, Pheng-Ann Hengb, Mubarak Shah, 2008,Shape matching and modeling using skeletal context, Pattern Recognition, 41 pp.1756 1767

Schmid,C.;Zisserman,A.,1997,Automatic line matching across views, IEEE Computer Society Conference on Computer Vision and Pattern Recognition,pp.666-671

S.Noronha ,R.Nevatia. 2001,Detection and modeling of building from multiple aerial images .IEEE Trans. PAMI,23,pp.501-518.

Tao Chen. 2006,Affine Invariant Feature Extraction of Images.Graduate School of National University of Defense Technology, Changsha, Hunan, P.R.China.pp.20-23.

Touzi R, Lopes A, Bousquet P. 1988,A statistical and geometrical edge detector for SAR images. IEEE Transactions on Geoscience and Remote Sensing, 26,pp.764-773.

Xin Kang,Chongzhao Han,Yi Yang. 2006,Automatic SAR Image Registration by Using Element Triangle Invariants, International Conference Information Fusion(ICIF '06),pp.203-207

Yasser Almehio;2010,Samia Bouchafa,Matching Images Using Invaria nt Level-

line Primitives under Projective Transformation,Canadian Conference o n Computer and Robot Vision, pp.130-135

Yosi Keller, Amir Averbach. 2003,Implicit similarity: a new approach to multi-sensor image registration. Proceedings of the 2003 IEEE Computer Society Conference on Computer Vision and Pattern Recognition. IEEE, pp.1-6,

Yosi Keller, 2006, Multisensor Image Registration via Implicit Similarity, IEEE transactions on pattern analysis and machine intelligence.,28.pp.794-801.

Zitova B, Flusser J . 2003,Image registration methods: A survey. Image and Vision Computing, 21,pp. 977-1000. 